\title{
Three Disk Oscillation Modes of Rotating Magnetized Neutron Stars
}

\author{
R.V.E. Lovelace \\ Departments of Astronomy and Applied and Engineering Physics, \\ Cornell University, Ithaca, NY 14853-6801; RVL1@cornell.edu \\ M.M. Romanova \\ Department of Astronomy, \\ Cornell University, Ithaca, NY 14853-6801; romanova@astro.cornell.edu
}

November 14, 2018

\begin{abstract}
We discuss three specific modes of accretion disks around rotating magnetized neutron stars which may explain the separations of the kilo Hertz quasi periodic oscillations (QPO) seen in low mass X-ray binaries. The existence of these modes requires that there be a maximum in the angular velocity of the accreting material, and that the fluid is in stable, nearly circular motion near this maximum rather than moving rapidly towards the star or out of the disk plane into funnel flows. It is presently not known if these conditions occur, but we are exploring this with 3D magnetohydrodynamic simulations and will report the results elsewhere. The first mode is a corotation mode which is radially trapped in the vicinity of the maximum of the disk rotation rate and is unstable. The second mode, relevant to relatively slowly rotating stars, is a magnetically driven eccentric $(m=1)$ oscillation of the disk excited at a Lindblad radius in the vicinity of the maximum of the disk rotation. The third mode, relevant to rapidly rotating stars, is a magnetically coupled eccentric $(m=1)$ and an axisymmetric $(m=0)$ radial disk perturbation which has an inner Lindblad radius also in the vicinity of the maximum of the disk rotation. We suggest that the first mode is associated with the upper QPO fre-
\end{abstract}

quency, $\nu_{u}$, the second with the lower QPO frequency, $\nu_{\ell}=\nu_{u}-\nu_{*}$, and the third with the lower QPO frequency, $\nu_{\ell}=\nu_{u}-\nu_{*} / 2$, where $\nu_{*}$ is the star's rotation rate.

keywords: accretion, accretion disks — stars: neutron - X-rays: binaries - magnetohydrodynamics

\section{Introduction}

Low mass X-ray binaries often display twin kiloHertz quasi-periodic oscillations (QPOs) in their Xray emissions (van der Klis 2006; Zhang et al. 2006). A wide variety of different models have been proposed to explain the origin and correlations of the different QPOs. These include the beat frequency model (Miller, Lamb, \& Psaltis 1998; Lamb \& Miller 2001; Lamb \& Miller 2003), the relativistic precession model (Stella \& Vietri 1999), the Alfvén wave model (Zhang 2004), and warped disk models (Shirakawa \& Lai 2002; Kato 2004).

A puzzling aspect of the some of the twin QPO sources considered in this work is that the difference between the upper $\nu_{u}$ and lower $\nu_{\ell}$ QPO frequencies is roughly either the spin frequency of the star $\nu_{*}\left(3\right.$ cases where $\left.\nu_{*}=270,330, \& 363 \mathrm{~Hz}\right)$ or one-half this frequency, $\nu_{*} / 2$ (4 cases where $\nu_{*}=$ $401,524,581, \& 619 \mathrm{~Hz})$, for the cases where $\nu_{*}$ 
is known, even though $\nu_{u}$ and $\nu_{\ell}$ vary significantly (see, e.g., Zhang et al. 2006). A further type of behavior appears in the source Cir X-1 (Boutloukos et al. 2006), but this is not considered here. The cases where $\nu_{u}-\nu_{\ell} \approx \nu_{*}$ may be explained by the beat frequency model (Miller et al. 1998), but the explanation of the cases where $\nu_{u}-\nu_{\ell} \approx \nu_{*} / 2$ is obscure.

Section 2.1 discusses the corotation instability, $\S 2.2$ the exccentric $(m=1)$ mode of the disk driven by the star's rotating magnetic field, and $\S 2.3$ the coupled exccentric plus axisymmetric mode $(m=0 \& 1)$ also due to the star's rotating magnetic field. Section 4 gives the conclusions.

\section{Three Modes}

\subsection{Corotation Instability}

We assume a pseudo-Newtonian gravitational potential $\Phi_{g}=-G M_{*} /\left(r-r_{S}\right)$, where $M_{*}$ is the star's mass and $r_{S} \equiv 2 G M_{*} / c^{2}$. In the absence of the star the angular velocity of disk matter is $\Omega_{g \phi}=$ $\left\{G M_{*} /\left[r\left(r-r_{S}\right)^{2}\right]\right\}^{1 / 2}$ for $r \geq 3 r_{S}$. Near the star the disk's angular rotation rate in the equatorial plane is modeled as

$$
\Omega_{\phi}(r)=\frac{\Omega_{*} f(r)}{1+f(r)}+\frac{\Omega_{g \phi}(r)}{1+f(r)},
$$

where $f(r)=\exp \left[-\left(r-r_{0}\right) / \Delta\right]$ with $r_{0}$ the standoff distance of the boundary layer and $\Delta$ its thickness which are expected to depend on the accretion rate and the star's magnetic field. The radial force equilibrium in the midplane of an axisymmetric disk is $\rho r\left(\Omega_{\phi}^{2}-\Omega_{g \phi}^{2}\right)=d\left(p+B^{2} / 8 \pi\right) / d r$, where $\rho$ is the midplane density and midplane field $\mathbf{B}=B(r) \hat{\mathbf{z}}$. Figure 1 shows the equilibrium quantities for an illustrative case. Clearly, $\Omega_{\phi}(r)$ has a maximum value outside of the star at a distance denoted $r_{m} \sim r_{0}$. The importance of this maximum for models of QPOs was discussed earlier by Alpar and Psaltis (2005). Three dimensional magnetohydrodynamic (MHD) simulations of disk accretion to rotating magnetized stars can in principle be used to determine $\Omega_{\phi}(r)$ for different conditions (Romanova, Kulkarni, \& Lovelace 2007). However, for the present purposes the dependence of equation (1) is used. It is needed only for distances $r \gtrsim r_{m}$ as discussed below. In this region the radial epicyclic frequency is $\Omega_{r}(r)=\left[r^{-3} d\left(r^{4} \Omega_{\phi}^{2}\right) / d r\right]^{1 / 2}$. The maximum value of $\Omega_{\phi}(r)$ has the approximate dependence $\max \left(\Omega_{\phi} / 2 \pi\right) \approx 2040\left(3 r_{S} / r_{0}\right)^{1.74} \mathrm{~Hz}$ for $3<r_{0} / r_{S}<5, \Delta / r_{S}=0.05$, and $M_{*}=1.4 M_{\odot}$. For this range of $r_{0}, \max \left(\Omega_{\phi}\right)$ changes by a factor of 2.4 .

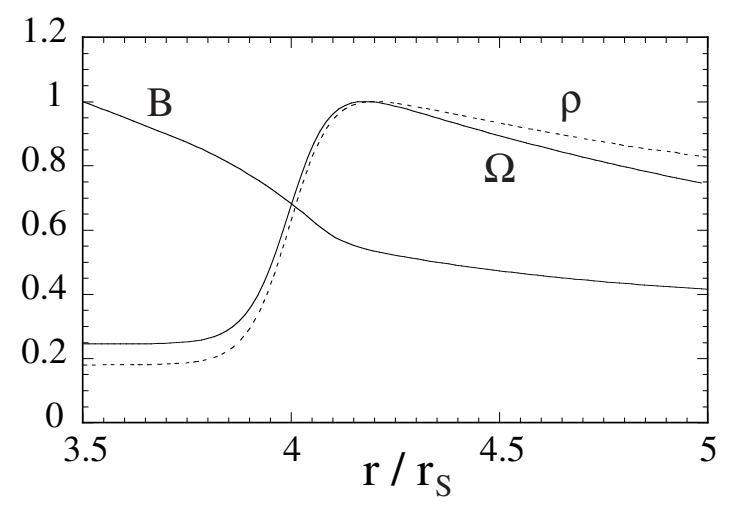

Figure 1: Equilibrium profiles of the main variables normalized to their maximum values. For this case, $M_{*}=1.4 M_{\odot}, \nu_{*}=\Omega_{*} / 2 \pi=300 \mathrm{~Hz}, r_{S} \approx 4.14 \times 10^{5}$ $\mathrm{cm}, r_{0}=4 r_{S}, \Delta=0.05 r_{S}, c_{s}=0.1 r \Omega_{\phi}, \rho_{\max } \approx$ $0.024 \mathrm{~g} / \mathrm{cm}^{3}, B\left(r / r_{S}=3.5\right) \approx 1.7 \times 10^{9} \mathrm{G}$, and $\max \left(\Omega_{\phi} / 2 \pi\right) \approx 1220 \mathrm{~Hz}$.

We consider a WKB treatment of the corotation $\left(\omega \approx m \Omega_{\phi}\right)$ or Rossby type wave of the disk with pressure perturbation

$$
\delta p \sim \exp \left(i \int^{r} d r k+i m \phi-i \omega t\right)
$$

where $k$ is the radial wavenumber, $m=1,2, .$. , and $\omega=\omega_{r}+i \omega_{i}$, with $\omega_{r}$ the angular frequency of the perturbation and $\omega_{i}$ the growth rate. For the conditions of Figure 1, the Alfvén speed $c_{A}=B /(4 \pi \rho)^{1 / 2}$ is much larger than the sound speed $c_{s}$. Also, we assume and verify later that $\left|\omega-m \Omega_{\phi}\right|^{2} \ll \Omega_{r}^{2}$. Under these conditions 
$k^{2}(r)=-\left(\frac{m}{r}\right)^{2}-\left(\frac{\Omega_{r}}{c_{A}}\right)^{2}-\frac{1}{L_{B}^{2}}-\frac{\Omega_{\phi}}{r \mathcal{F}} \frac{d}{d r}\left(\frac{r \mathcal{F}}{\Omega_{\phi} L_{B}}\right)$

$-\frac{2 m}{r}\left(\frac{2}{L_{B}}+\frac{1}{\mathcal{F}} \frac{d \mathcal{F}}{d r}\right) \Re\left(\frac{\Omega_{\phi}}{\Delta \omega}\right)-\left(\frac{m}{r}\right)^{2} \frac{c_{A}^{2}}{L_{B} L_{b}} \Re\left(\frac{1}{\Delta \omega^{2}}\right)$

where $\Delta \omega \equiv \omega-m \Omega_{\phi}, L_{B}^{-1} \equiv d \ln (B / \rho) / d r, L_{b}^{-1} \equiv$ $d \ln (B) / d r, \mathcal{F} \equiv \rho \Omega_{\phi} / \Omega_{r}^{2}$, which all depend on $r$, and $\Re(.$.$) denotes the real part (Lovelace, Turner, \& Ro-$ manova 2007). Equation (2) generalizes the calculation of Lovelace et al. (1999) to include the influence of the magnetic field perturbation $\delta \mathbf{B}=\delta B(r, \phi, t) \hat{\mathbf{z}}$.

Figure 2 shows the radial dependence of $k^{2}$ for a representative case. For the chosen value $\omega_{r} / 2 \pi=$ $1100 \mathrm{~Hz}$, which is somewhat less than the maximum value of $\Omega_{\phi}(r)$, and $m=1$, it is seen that $k^{2}(r) \geq 0$ in a finite radial interval in the vicinity of the maximum of $\Omega_{\phi}(r)$. Thus the wave is radially trapped in the vicinity of the maximum of $\Omega_{\phi}(r)$. Analogous radially trapped modes were analyzed earlier by Lovelace et al. (1999) and $\mathrm{Li}$ et al. (2000) and verified in two dimensional hydrodynamic simulations ( $\mathrm{Li}$ et al. 2001). The Bohr-Sommerfeld quantization condition $\int_{r \text { (in) }}^{r \text { (out) }} d r k=(n+1 / 2) \pi, n=0,1, .$. allows the determination of the growth rate $\omega_{i}$. For the case shown, $\omega_{i} / 2 \pi=\nu_{i} \approx 55 \mathrm{~Hz}$ for $n=0$ which gives the largest growth rate, and $r($ in $) / r_{S}=4.08, r$ (out) $/ r_{S}=4.48$. The growth rate increases as $\Delta$ decreases. Similar values of the growth rates are found for $\omega_{r}$ somewhat less than $m \max \left(\Omega_{\phi}\right)$ for $m=2,3, \ldots$ The nonlinear saturation of the growth of the modes can in principle be found by MHD simulations (Koldoba et al. 2002; Romanova et al. 2007).

From Figure 2 we see that the validity of equation (1) is needed only from the vicinity of the maximum of $\Omega_{\phi}(r)$, that is, from $r / r_{S}=4.08$ where $\Omega_{\phi} / \max \left(\Omega_{\phi}\right)=0.94$ (the inner turning point) and outward (including the outer turning point at $r / r_{S}=$ 4.48).

Owing to the perturbation, the surface temperature of the disk is $T(r, \phi, t)=$

$T_{0}+\delta T_{1} \exp \left(i \phi-\omega_{1} t\right)+\delta T_{2}(r) \exp \left(2 i \phi-i \omega_{2} t\right)+\ldots$, where $T_{0}(r)$ is the unperturbed temperature, $\delta T_{1,2}(r) \ll T_{0}$ are the amplitudes of the $m=1,2$ corotation modes, and $\omega_{1,2}$ are their frequencies. The corresponding flux density is proportional to $S(r, \phi, t) \sim T_{0}^{4}+4 T_{0}^{3} \delta T_{1} \exp \left(i \phi-\omega_{1} t\right)+.$. The toal flux for a face-on disk, $L \sim \int r d r d \phi S$, is independent of time. For a more general disk orientation, the Doppler effect due to the disk rotation gives a boost for say $\phi=0$ and a decrement for $\phi=\pi$. This corresponds to multiplying $S$ by $[1+\epsilon \exp (-i \phi)]$, with $\epsilon(r) \ll 1$. Consequently, there is a contribution to the source flux $\delta L \sim \int r d r d \phi \exp (-i \phi) S(r, \phi, t) \sim$ $4 \int r d r T_{0}^{3} \epsilon \delta T_{1}(r) \exp \left(-i \omega_{1} t\right)$. As explained in the next section, we interpret this frequency as the upper frequency component of the twin QPOs.

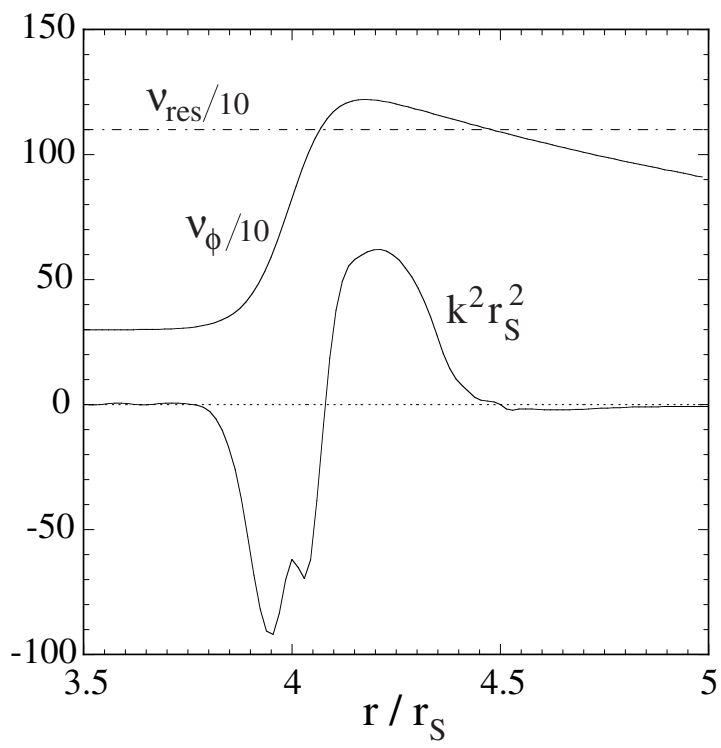

Figure 2: Radial dependences of the different frequencies $\nu_{\phi}=\Omega_{\phi} / 2 \pi$, the resonant frequency $\nu_{r e s}=$ $\omega_{r} / 2 \pi=1100 \mathrm{~Hz}$, and the square of the radial wavenumber, $k^{2}$ obtained from equation (2) for $m=$ 1 and for the same conditions as for Figure 1. 


\subsection{Magnetically Driven $m=1$ Mode}

We now give a heuristic treatment of perturbations of the disk excited by the star's rotating, nonaxisymmetric magnetic field. One component of a general perturbation is described by the radial displacement of the disk matter, $\mathcal{E}(r, \phi, t)$ (e.g., Zhang $\&$ Lovelace 2005) The equation for $\mathcal{E}$ is simply

$$
\frac{d^{2} \mathcal{E}}{d t^{2}}=-\left(\Omega_{r}^{2}+k^{2} c_{s}^{2}\right) \mathcal{E}+\delta F(r, \phi, t)
$$

where $d / d t \equiv \partial / \partial t+\Omega_{\phi} \partial / \partial \phi$, and $\delta F$ is the radial force due to the star's magnetic field. The radial oscillation frequency (squared) on the right-hand side of the equation consists $\Omega_{r}^{2}$ as given earlier plus a thermal contribution $k^{2} c_{s}^{2}$, where $k$ is the radial wavenumber of the perturbation in the WKB approximation.

We consider the case where the star has a small size or point-like dipole located close to the star's surface near the rotation axis with its magnetic moment parallel to the star's surface. Ruderman (2006) gives physical arguments for this type of configuration of spun-up neutron stars. At a given radial distance in the equatorial plane, the total magnetic field $B_{z}=B_{z}^{v}+B_{z}^{i}$ consists of the vacuum component $B_{z}^{v}=B_{0}(r) \cos \left(\phi-\Omega_{*} t\right)$, with $\Omega_{*}$ the star's angular rotation rate, and the induced component, $B_{z}^{i}$. The component $B_{z}^{v}$ acts to drive a current flow in the disk $\mathcal{K}_{\phi}$ which in turn gives rise to the induced magnetic field of the disk $B_{z}^{i}$. The radial force includes a coherent component $\propto B_{z}^{v}$ which can be written as $\delta F=C_{1} \exp \left(i \phi-i \Omega_{*} t\right)$, where $C_{1}(r)=\left\langle\mathcal{K}_{\phi}\right\rangle B_{0} /(c \Sigma)$ with the average is over $\phi$. Considering only the coherent component, equation (4) is then a driven oscillator. With $i k \rightarrow d / d r$ and $\mathcal{E} \sim \exp \left(i \phi-i \Omega_{*} t\right)$, it becomes

$$
\left(\frac{d^{2}}{d r^{2}}-\mathcal{D}(r)\right) \mathcal{E}=-C_{1} / c_{s}^{2},
$$

with $\mathcal{D} \equiv\left[\Omega_{r}^{2}-\left(\Omega_{\phi}-\Omega_{*}\right)^{2}\right] / c_{s}^{2}$. Depending mainly on the value of $\Omega_{*}$, there may be Lindblad resonances with inner and outer Lindblad radii $r_{L i, o}$ where $\mathcal{D}\left(r_{L i, o}\right)=0$. The region between the two radii is permitted in the sense that $\mathcal{D} \leq 0$ while the regions $r>r_{L o}$ and $r<r_{L i}$ are forbidden. The existence of the Lindblad resonances means that a weak magnetic disturbance can give rise to a strong disk response in the vicinity of $r_{L}$ proportional to $C_{1}\left(r_{L}\right)$ (Goldreich \& Tremaine 1979). Because of the rapid decrease of the magnetic field the response at the inner Lindblad resonance is expected to be stronger than that at the outer resonance. However, the solution of equation (5) is beyond the scope of this work. We find that there are Lindblad resonances only for for $\nu_{*}=\Omega_{*} / 2 \pi<380 \mathrm{~Hz}$ for the same conditions as Figure 2. For higher $\nu_{*}$, all values of $r$ are forbidden. For $\nu_{*}=300 \mathrm{~Hz}, r_{L i} / r_{S} \approx 4.34$, which is within the region of the mentioned corotation instability, and $r_{L o} / r_{S} \approx 4.83$ which is outside the region of corotation instability. The disk velocity $\Omega_{\phi} r$ is supersonic relative to the velocity of the perturbation $\Omega_{*} r$ for $r$ larger than $r_{L i}$. Consequently the perturbation is a leading spiral wave with $k<0$. At the outer Lindblad radius the disk velocity is also supersonic relative to $\Omega_{*} r$, and excitation of the disk at this radius gives a trailing spiral, $k>0$ for $r<r_{L o}$.

The interaction between the corotation perturbation and the magnetic perturbation of the disk is in general nonlinear. The perturbed disk surface temperature can be represented as a product of the two perturbations $\tilde{T}(r, \phi, t) \sim[1+$ $\left.\epsilon_{M}|\mathcal{E} / r| \exp \left(-i \phi+i \Omega_{*} t\right)\right]\left[T_{0}+\delta T_{1} \exp \left(i \phi-i \omega_{1} t\right)+\right.$ $..]=T_{0}+\epsilon_{M}|\mathcal{E} / r| \delta T_{1} \exp \left[-i\left(\omega_{1}-\Omega_{*}\right) t\right]+.$. , where $\epsilon_{M} \leq 1$. Consequently, there is a contribution to the source flux $\delta L \sim \int r d r d \phi \exp (-i \phi) S(r, \phi, t) \sim$ $4 \int r d r T_{0}^{3} \epsilon_{M}|\mathcal{E} / r| \delta T_{1}(r) \exp \left[-i\left(\omega_{1}-\Omega_{*}\right) t\right]$, where $S \sim$ $\tilde{T}^{4}$. For $\nu_{*}<\sim 380 \mathrm{~Hz}$, we interpret $\omega_{1}-\Omega_{*}$ as the lower frequency component of twin QPOs.

\subsection{Magnetically Coupled $m=0 \& 1$ Mode}

Consider now higher rotation frequencies $\nu_{*}$. Note that the radial force perturbation includes a contribution of the form $\delta F \sim \Sigma^{-1} \Re(\mathcal{E}) \partial\left(\mathcal{K}_{\phi} B_{z} / c\right) / \partial r$ which again has a coherent term proportional to $B_{z}^{v}$. We consider only the coherent term which can be written as $\delta F=\Re(\mathcal{E}) D_{1} \cos \left(\phi-\Omega_{*} t\right)$, where $D_{1}=\partial\left\langle\mathcal{K}_{\phi} B_{0}\right\rangle / \partial r /(c \Sigma)=$ real. In this case $\mathcal{E}$ necessarily consists of different $\phi$-harmonics. That is, $\mathcal{E}=$ $\mathcal{E}_{0} \exp (-i \omega t)+\mathcal{E}_{1} \exp (i \phi-i \omega t)+\mathcal{E}_{2} \exp (2 i \phi-\omega t)+\ldots$ 
We find

$$
\begin{aligned}
\left(c_{s}^{2} \frac{d^{2}}{d r^{2}}-\left[\Omega_{r}^{2}-\omega^{2}\right]\right) \mathcal{E}_{0} & =-\frac{1}{2} \mathcal{E}_{1}^{*} D_{1}, \\
\left(c_{s}^{2} \frac{d^{2}}{d r^{2}}-\left[\Omega_{r}^{2}-\left(\Omega_{\phi}-\omega\right)^{2}\right]\right) \mathcal{E}_{1} & =-\frac{1}{2} \mathcal{E}_{0}^{*} D_{1}
\end{aligned}
$$

where necessarily $\omega=\Omega_{*} / 2$. For the radii of interest in the vicinity of $r_{0}, \Omega_{r}^{2} \gg \omega^{2}$, so that the righthand side of the second equation is $\approx-D_{1}^{2} \mathcal{E}_{1} /\left(4 \Omega_{r}^{2}\right)$. We identify $\left|D_{1}(r)\right|$ as the Alfvén frequency squared $\Omega_{A}^{2}$ at the distance $r$ and estimate that $\Omega_{A}^{2} \ll \Omega_{r}^{2}$. Evidently the equation for $\mathcal{E}_{1}$ corresponds to free oscillations. It has Lindblad radii approximately where $\mathcal{D}(r) \equiv \Omega_{r}^{2}-\left(\Omega_{\phi}-\omega\right)^{2}=0$. For $\nu_{*}=\omega / \pi=600$ $\mathrm{Hz}$ and the other parameters the same as in Figure 2, the inner Lindblad radius is at $r_{L i} / r_{S}=4.2$ while the outer Lindblad radius is at a large distance $\left(r / r_{S}>6\right)$. This mode may be driven at $r_{L i}$ by noise or fluctuations in the disk at this radius, and it is a leading spiral wave. The mode amplitude can in principle be found using three-dimensional MHD simulations (Koldoba, et al. 2002; Romanova et al. 2007).

The influence of the magnetically coupled modes on the flux follows the discussion of $\$ 2.3$. We find $\delta L \sim \int r d r d \phi \exp (-i \phi) S(r, \phi, t) \sim$ $4 \int r d r T_{0}^{3} \epsilon_{M}\left|\mathcal{E}_{1} / r\right| \delta T_{1}(r) \exp \left[-i\left(\omega_{1}-\Omega_{*} / 2\right) t\right]$. For $\nu_{*}>\sim 380 \mathrm{~Hz}$, we interpret $\omega_{1}-\Omega_{*} / 2$ as the lower frequency component of twin QPOs.

\section{Conclusions}

We discuss three modes of accretion disks around rotating magnetized neutron stars which may explain the frequency separations of the twin kilo-Hertz QPOs seen in accreting X-ray binaries. The existence of these modes requires that there be a maximum in the angular velocity of the accreting material and that the fluid is in stable, nearly circular motion near this maximum rather than moving rapidly towards the star or out of the disk plane into funnel flows. It is presently not known if these conditions occur, but we are exploring this with 3D magnetohydrodynamic simulations and will report the results elsewhere. The first mode is a corotation mode which is radially trapped in the vicinity of the maximum of the disk rotation rate and is unstable. A simple dependence is assumed for the angular rotation rate of the disk $\Omega_{\phi}(r)$ which has a maximum at a radius $r_{m}$ outside the neutron star. The unstable mode has a frequency $\omega_{1}$ somewhat less than $\max \left(\Omega_{\phi}\right)$ and this can vary by a significant factor depending on the state of the disk (e.g., the accretion rate). We suggest that this mode is associated with the upper QPO frequency, $\nu_{u}=\omega_{1} / 2 \pi$. The second mode is a magnetically driven eccentric $(m=1)$ oscillation of the disk excited at the inner Lindblad radius which is in the vicinity of the maximum of the disk rotation. The star's magnetic field is assumed to be the form discussed by Ruderman (2006). The Lindblad radii occur only for relatively slowly rotating stars, $\nu_{*}<\sim 380 \mathrm{~Hz}$. For these stars we suggest that the lower QPO frequency is $\nu_{\ell}=\nu_{u}-\nu_{*}$. The third mode, relevant to more rapidly rotating stars, is a magnetically coupled eccentric $(m=1)$ and an axisymmetric $(m=0)$ radial disk perturbation. It has an inner Lindblad radius also in the vicinity of the maximum of the disk rotation. For these stars the lower QPO frequency is $\nu_{\ell}=\nu_{u}-\nu_{*} / 2$. A problem remaining for future work is the determination of the saturation amplitudes of the different modes.

\section{Acknowledgements}

We thank an anonymous referee for valuable criticism of an earlier version of this work. This work was supported in part by NASA grants NAG5-13220 and NAG5-13060 and by NSF grant AST-0507760.

\section{References}

[1] Alpar, M.A., \& Psaltis, D. 2005, astro-ph/0511412

[2] Boutloukos, S., van der Klis, M., Altamirano, D., Klein-Wolt, M., Wijnands, R., Jonker, P.G., \& Fender, R.P. 2006 ApJ, 653, 1435

[3] Goldreich, P., \& Tremaine, S. 1979, ApJ, 233, 857

[4] Kato, S. 2004, PASJ, 56, 905 
[5] Koldoba, A.V., Romanova, M.M., Ustyugova, G.V., \& Lovelace, R.V.E. 2002, ApJ, 576, L53

[6] Lamb, F.K., \& Miller, M.C. 2001, ApJ, 554, 1210

[7] Lamb, F.K., \& Miller, M.C. 2003, astro-ph/0308179

[8] Li, H., Finn, J.M., Lovelace, R.V.E., \& Colgate, S.A. 2000, ApJ, 533, 1023

[9] Li, H., Colgate, S.A., Wendroff, B., \& Liska, R. 2001, ApJ, 551, 874

[10] Lovelace, R.V.E., Li, H., Colgate, S.A., \& Nelson, A.F. 1999, ApJ, 513, 805

[11] Lovelace, R.V.E., Turner, L., \& Romanova, M.M. 2007, in prepration

[12] Miller, M.C., Lamb, F.K., \& Psaltis, D. 1998, ApJ, 508, 791

[13] Romanova, M.M., Kulkarni, A.K., \& Lovelace, R.V.E. 2007, ApJ, submitted

[14] Ruderman, M. 2006, astro-ph/0610375

[15] Shirakawa, A., \& Lai, D. 2002, ApJ, 564, 361

[16] Stella, L., \& Vietri, M. 1999, Phys. Rev. Lett., 82,17

[17] van der Klis, M. 2006, in Compact Stellar XRay Sources, Eds. W.H.G. Lewin \& M. van der Klis (Cambridge: Cambridge Univ. Press), p. 39

[18] Zhang, C. 2004, A\&A, 423, 401

[19] Zhang, C.M., Yin, H.Z., Zhao, Y.H., Zhang, F., \& Song, L.M. 2006, MNRAS, 366, 1373

[20] Zhang, L., \& Lovelace, R.V.E. 2005, Ap\&SS, 300,385 